TITLE:

\title{
Nucleotide Sequence of cDNA and the Gene Expression of Testis- Specific Protein $Y$ in the Japanese Monkey
}

\section{AUTHOR(S):}

Kim, Heui-Soo; Kageyama, Takashi; Nakamura, Shin; Takenaka, Osamu

\section{CITATION:}

Kim, Heui-Soo ... [et al]. Nucleotide Sequence of cDNA and the Gene Expression of TestisSpecific Protein Y in the Japanese Monkey. Zoological Science 1997, 14(4): 609-614

\section{ISSUE DATE:}

1997-04

URL:

http://hdl.handle.net/2433/108624

\section{RIGHT:}

(c) 日本動物学会 / Zoological Society of Japan 


\title{
Nucleotide Sequence of cDNA and the Gene Expression of Testis-Specific Protein $\mathrm{Y}$ in the Japanese Monkey
}

\author{
Heui-Soo Kim, Takashi Kageyama, Shin Nakamura \\ and Osamu Takenaka* \\ Department of Cellular and Molecular Biology, Primate Research Institute, \\ Kyoto University, Inuyama, Aichi 484, Japan
}

\begin{abstract}
We cloned the cDNA for Japanese monkey Testis-Specific Protein Y (TSPY). The cDNA contained an open reading frame of 246 amino acids. This coding fragment shared $89 \%$ nucleotide sequence identity and $81 \%$ amino acid sequence identity with the homologous fragment of previously isolated human TSPY CDNA. Monkey TSPY was assumed to be a molecular mass of $28 \mathrm{kDa}$ and an isoelectric point of $\mathrm{pH}$ 5.35. This protein was hydrophilic and contained an Arg and Lys-rich region which was a potential DNA binding site. Expression of the TSPY gene examined by reverse transcription PCR showed that the transcript was detectable only in testis, suggesting that TSPY plays an important role in spermatogenesis of primates.
\end{abstract}

\section{INTRODUCTION}

Testis-specific protein $Y$ (TSPY) is known to be a product of the $\mathrm{Y}$-chromosome specific gene. The expression of the TSPY gene has been shown to be restricted to testicular tissue (Arnemann et al., 1991) and appeared to be confined to germ cells of the spermatogonial and early spermatocyte stages in adult human males (Chandley and Cooke, 1994; Schnieders et al., 1996). Although the exact function of TSPY is still unknown, this protein might play a role in DNA replication (Schnieders et al., 1996). A cDNA clone for human TSPY was isolated from an adult human testis CDNA library (Arnemann et al., 1991). A genomic clone for human TSPY was also isolated successively (Zhang et al., 1992). The gene contained six exons with five introns and was estimated to be approximately $2.7 \mathrm{~kb}$.

Human TSPY gene-related sequences are organized as constitutive parts of DYZ5 repeat units (Manz et al., 1993) which are located on the short arm of the $Y$ chromosome (TylerSmith et al., 1988). DYZ5 sequences have been shown to be conserved on the $Y$ chromosome of the great apes by Southern blot and in situ hybridization (Guttenbach et al., 1992). Using the chromosomal in situ hybridization technique, Schempp et al. (1995) showed that TSPY gene-related sequences are conserved and $Y$ chromosome specific in hominoids. The number of TSPY genes and related genes are highly amplified especially in primates (Kim and Takenaka, 1996; Kim et al., 1996). We have sequenced exon 1, exon 2 , and the first intron of the TSPY gene of great apes and the baboon and determined phylogenetic relationship among them

* Corresponding author: Tel. +81-568-63-0577;

FAX. +81-568-62-9557.
(Kim and Takenaka, 1996). In our succeeding report, we compared restriction patterns and chromosomal localizations of TSPY genes in man, gibbons, and Old World monkeys, and found variations of gene structures among them (Kim et al., 1996). Since in primates it is possible that TSPY genes evolve some structural differentiations and cause specificities in reproductive systems, it is necessary to clarify the structures of TSPY genes in various primates. To date, however, the complete structure of a TSPY gene is not known except for the human gene.

In the present study, we cloned and sequenced a fulllength of the TSPY CDNA from Japanese monkey testis RNA, described its molecular characterizations, and examined the expression of the TSPY gene in various tissues.

\section{MATERIALS AND METHODS}

\section{Isolation of total RNA from monkey tissues}

Tissues were collected from a 6-year-old Japanese monkey (Macaca fuscata) immediately after death by exsanguination via bilateral carotid arteries under deep anesthesia with ketamine hydrochloride and sodium pentobarbital, in accordance with the guideline of the Primate Research Institute, Kyoto University. Total RNA was extracted by $T R I Z O L$ reagent $(B R L)$.

\section{Cloning of the cDNA for Japanese monkey TSPY}

Full-length TSPY cDNA was prepared by RT-PCR. First, TSPY mRNA was reverse transcribed into single-stranded CDNA by AMV reverse transcriptase (BRL) using a primer, P356 (5'-CCTTGAGAATGTTTATTTTTCATTCC-3'). Following this synthesis, cDNA was amplified by PCR using primers P356 and P459 (5'-CCAAGGAGGGCACCGCCTTC-3'). Primers P356 and P459 were designed based on published sequences of human TSPY cDNA (Zhang et al., 1992). Their locations in TSPY cDNA are shown in Fig. 1. The PCR was performed with a thermocycler manufactured by Perkin Elmer Cetus (Model 9600) as follows. After the initial denaturation step at $94^{\circ} \mathrm{C}$ for $3 \mathrm{~min}$, DNA 


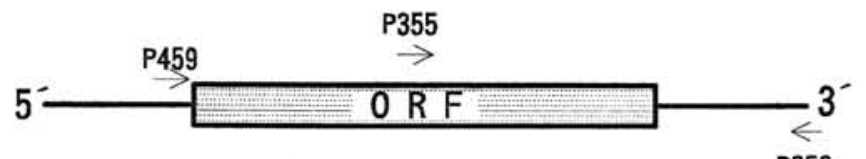

P356

Fig. 1. Primer locations for RT-PCR. All primers were designed based on published sequences of human TSPY gene. The open box represents the open reading frame (ORF).

was amplified for 40 cycles at $94^{\circ} \mathrm{C}$ for $1 \mathrm{~min}, 60^{\circ} \mathrm{C}$ for $1 \mathrm{~min}$ and $72^{\circ} \mathrm{C}$ for $1.5 \mathrm{~min}$. The $\mathrm{PCR}$ products were analyzed with agarose gel electrophoresis, purified by QIAEX gel extraction kit (QIAGEN), and cloned into $\mathrm{T}$-vector which was prepared by modification of the Holton and Graham (1991) method.

\begin{abstract}
Sequencing and data analyses
The nucleotide sequence was determined on both strands of the insert DNA using the dideoxy chain termination method (Sanger et al., 1977). At least three cloned fragments from each of the PCRamplified DNAs were sequenced. The various analyses of nucleotide sequences and encoded amino acid sequences were done with the aid of the GENETYX system (Ver. 9, SDC, Tokyo). Sequence similarity was searched for in the protein database from SWISS-PRO and the hydropathy value was calculated using the method of Eisenberg et al. (1984). The pairwise distance of the number of nucleotide substitutions was estimated using the method of Tajima and Nei (1984). The number of synonymous and nonsynonymous nucleotide substitutions were obtained by the MEGA program (Ver. 1.01, USA).
\end{abstract}

\section{Detection of TSPY mRNA by RT-PCR}

Expression of the TSPY gene in various tissues was analyzed by RT-PCR with specific primers P355 (5'-CAGATGTCAGCCCTGATCACTG-3') and P356 (5'-CCTTGAGAATGTTTATTTTTCATTCC-3') using Taq DNA polymerase. The locations of these primers in TSPY cDNA are shown in Fig. 1. The size of the PCR product was expected to be $630 \mathrm{bp}$. As a standard control for this method, we also examined the expression of the human G3PDH gene with the primers GPDS (5'-ACCACAGTCCATGCCATCAC-3') and GPD-AS (5'-TCCACCACCCTGTTGCTGTA-3') using $r$ Tth DNA polymerase (TOYOBO). The parameters included $1 \mathrm{cycle}$ at $60^{\circ} \mathrm{C}$ for $30 \mathrm{~min}$, followed by $94^{\circ} \mathrm{C}$ for $2 \mathrm{~min}$, then 40 cycles at $94^{\circ} \mathrm{C}$ for $1 \mathrm{~min}$ and $60^{\circ} \mathrm{C}$ for $1.5 \mathrm{~min}$, with an additional step of $60^{\circ} \mathrm{C}$ for $7 \mathrm{~min}$. The PCR products were electrophoresed on $1.5 \%$ agarose gel, stained with ethidium bromide, and photodocumented on a UV transilluminator.

\section{RESULTS AND DISCUSSION}

\section{Molecular cloning of TSPY cDNA and structure analysis}

The nucleotide and deduced amino acid sequences of monkey TSPY cDNA are shown in Fig. 2. The nucleotide sequences were the same in three independent clones of RTPCR products, being almost certain that the sequence was free from the mutated ones during amplification. The cDNA consisted of 976 nucleotides, which included an open reading frame of 741 nucleotides encoding 246 amino acids. The ATG triplet indicated as nucleotides 1-3 was an initiation codon of the open reading frame and stop codon appeared in nucleotides $739-741$ as a TGA triplet. A polyadenylation site, AATAAA, was not included in the sequence since we used an oligonucleotide containing this site as a PCR primer. The analysis of the deduced amino acid sequence indicates that the gene product is a slightly acidic protein with an isoelectric point of $\mathrm{pH} 5.35$ and has a molecular mass of $28 \mathrm{kDa}$. Amino acids contained at high levels in monkey TSPY were Glu (10.6\%), Ala $(9.4 \%)$, and $\operatorname{Arg}(7.3 \%)$. The sum of Glu and Asp was higher than that of basic residues such as Arg and Lys being consistent with the monkey TSPY which was an acidic protein. One of the characteristic features of this protein was its hydrophilic nature, and three hydrophilic regions (residues 42 51, 79-105, and 202-207) were clearly identified (Fig. 3). Interestingly, the 26-residue segment (residues 79-105) had an abundance of basic residues such as Arg and Lys. The region was well conserved between monkey, human, and bovine TSPY, and positively charged residues were concentrated in the lined box (Fig.5). This finding allows us to speculate that these residues may be implicated in the DNA binding property as a structural evidence of the Arg and Lys-rich region. Such a feature was already appeared in the high mobility group (HMG) box, a main functional domain, of the sex determining region Y (SRY) (Jantzen et al., 1990). The putative nucleicacid-binding motif within SRY and its testis specific expression is consistent with that of SRY which has a role in the developmental regulation of the testis (Sinclair et al., 1990). TSPY may represent such a function in monkey testis. The Arg and Lys-rich sequence might have other roles such as that serving as a nuclear localization signal (Schnieders et al., 1996). The exact function of this characteristic sequence remains to be clarified.

\section{Comparison of TSPY sequences}

The similarity of nucleotide sequences between monkey and human TSPY CDNAs was $85.7 \%$ as a whole, and $88.6 \%$ and $76.3 \%$ in the coding and $3^{\prime}$ untranslated regions, respectively. The deduced amino acid sequence of monkey TSPY CDNA revealed an identity of $81.4 \%$ with the human TSPY (Fig. 5). Twenty-one deletions in nucleotide sequence (deletion of 7 amino acids in protein sequence) in the monkey coding region and eighteen insertions in the monkey $3^{\prime}$ untranslated region were noted. The deleted sequence in monkey TSPY were corresponded to Pro-Arg-Glu at position 51-53 and Ser-Pro-Asp-Arg at position 234-237 of human TSPY. A fragment of bovine TSPY gene was isolated by Jakubiczka et al. (1993), and was shown to have high similarity $(74.8 \%)$ with the human TSPY gene. The corresponding nucleotide sequences in monkey TSPY cDNA were slightly higher in similarity $(78 \%)$ (Table 1). Amino acid sequences between monkey, human, and bovine TSPYs showed that the similarity between monkey and human TSPYS $(81.4 \%)$ were much higher than those between monkey and bovine TSPYS (57.5\%) (Fig. 5). Since nucleotide sequences between monkey and bovine TSPYs were showed high similarity, the high numbers of amino acid substitutions between them were caused by the high ratio of substitutions in the first or second codons between the triplets of the coding regions of monkey and bovine TSPY genes.

In order to clarify TSPY gene evolution, we calculated the pairwise distance of the number of nucleotide substitu- 


$\begin{array}{rrrrrrrrr}10 & 20 & 30 & 40 & 50 & 60 & 70 & 80 & 90\end{array}$

ATGGAGTCTCTACAGGAGGGGGAGGCCGGGGCGCAGAGCGAGCAGGTAGCTTTGGGGGAGGAGGCGGTGCTGGGAGCGGATGACATAATG

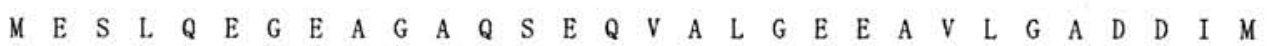
$\begin{array}{lllllllll}100 & 110 & 120 & 130 & 140 & 150 & 160 & 170 & 180\end{array}$ GCGGAGGTGGAGGTGGTGGCCCACCAGGAAGCCGACGAGAAGCGGCAGGAGCAGGTCCAGCGGGCACAGCCTGGCCCTGGGCCCATGAGC

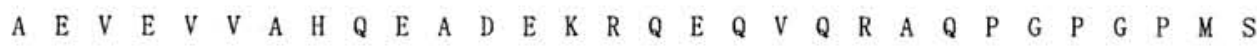
$\begin{array}{lllllllll}190 & 200 & 210 & 220 & 230 & 240 & 250 & 260 & 270\end{array}$ CCAGAGTCTGCACTGGAGGAGCTGCTGGCCGTTCAGGTGGAGCTGGAGCCGGTTAATTCCCGAGCCAGGAAGGCCTTTTCTCAGCAGAGG

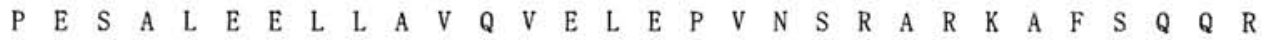
$\begin{array}{llllllll}280 & 290 & 300 & 310 & 320 & 330 & 340 & 350\end{array}$ GAAAAGATGGAGCGGAGGCGCAAGCCCCACCTGGACCGCAGAGGCGCCATCATCCAGAGCATGCCTGGCTTCTGGGCCAATGTTATTGCA

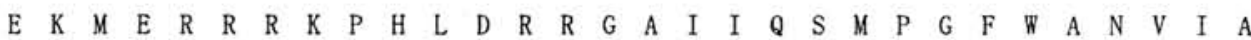
$\begin{array}{lllllllll}370 & 380 & 390 & 400 & 410 & 420 & 430 & 440 & 450\end{array}$ AACCACCCTCGGATGTCGGCCCTGCTCACTGACCAAGATGAAGACATGCTGAGCTACATGATCAACTTGGAGGTGAAAGAAGCGAAGCAT

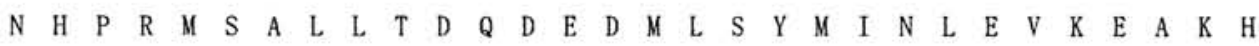
$\begin{array}{llllllll}460 & 470 & 480 & 490 & 500 & 510 & 520 & 530\end{array}$ CCCGTTCATCTCTGCCAGATCATGTTGTTCTTTCGGAGTAACCCCTACTTCCAGAATAAAGTGATTACCAAGGAATATCTCGTGAACGTC

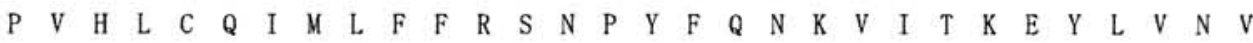
$\begin{array}{lllllllll}550 & 560 & 570 & 580 & 590 & 600 & 610 & 620 & 630\end{array}$ ACAGAATACAGGGCTTCTCATTCCACTCCAATTCAGTGGTGTCAGGATTATGAAGTTGAGGCCTATCGCCGCAGACACAACAACAGCGGT

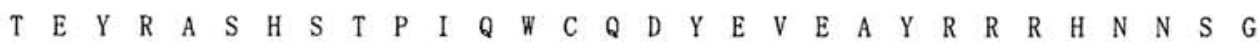
$\begin{array}{lllllllll}640 & 650 & 660 & 670 & 680 & 690 & 700 & 710 & 720\end{array}$ CTTAACTTCTTCAACTGGTTTTCTGACCACAACTTCGCAGGATCCAATAGGATTGCTGAGTCCTATGTAAGGACCTGTGGCGCAATCCCC

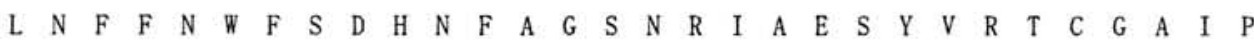
$\begin{array}{lllllllll}730 & 740 & 750 & 760 & 770 & 780 & 790 & 800 & 810\end{array}$ TGCAATACTACAGGAGGATGAAGCCACCTGAAGAGGAAACAGAGATTTCAGGGAACGCGCAGATGTTGGGTTGAATATGATGGAGCATCG $\begin{array}{lllllllllllllll}C & N & T & T & G & G & *\end{array}$ $\begin{array}{lllllllll}820 & 830 & 840 & 850 & 860 & 870 & 880 & 890 & 900\end{array}$ GACACAGGTGCGTGTTCACCTAACACGGCAGAACTCCTGAAAACTTACTACAGTATGCAGGATGTCAGCACTCAGCATGGTCTTGTGCAC $\begin{array}{lllllll}910 & 920 & 930 & 940 & 950 & 960 & 970\end{array}$

AGGAACTAAAGGACAAGCAGATCCAGTCACAGCAAATGAAGACAGGAAGCGGGGTGGGGGAATTGAATTGTAT

Fig. 2. Nucleotide sequences of the monkey TSPY CDNA (accession no. AB001421) together with the translation of its open reading frame. Amino acids are shown in single-letter codes. The initiation codon (ATG) is underlined. The termination codon (TGA) is indicated by an asterisk.

tions per site $\left(\mathrm{K}_{\mathrm{N}}\right)$ and the rate of nucleotide substitutions per site per year $\left(\mathrm{V}_{\mathrm{N}}\right)$ using divergence times from paleontological data (Gingerich, 1984; Pilbeam, 1986). As shown in Table 2 , the $K_{N}$ and $V_{N}$ values in the coding region between monkey and human TSPY cDNAs were 0.096 and 1.6-2.4 $\times 10^{-9} /$ site / year, respectively. Whereas, the $K_{N}$ and $V_{N}$ values in the $3^{\prime}$ untranslated region between them were 0.199 and 3.3-5.0 $\times$ $10^{-9} /$ site / year, respectively. These values are almost the 


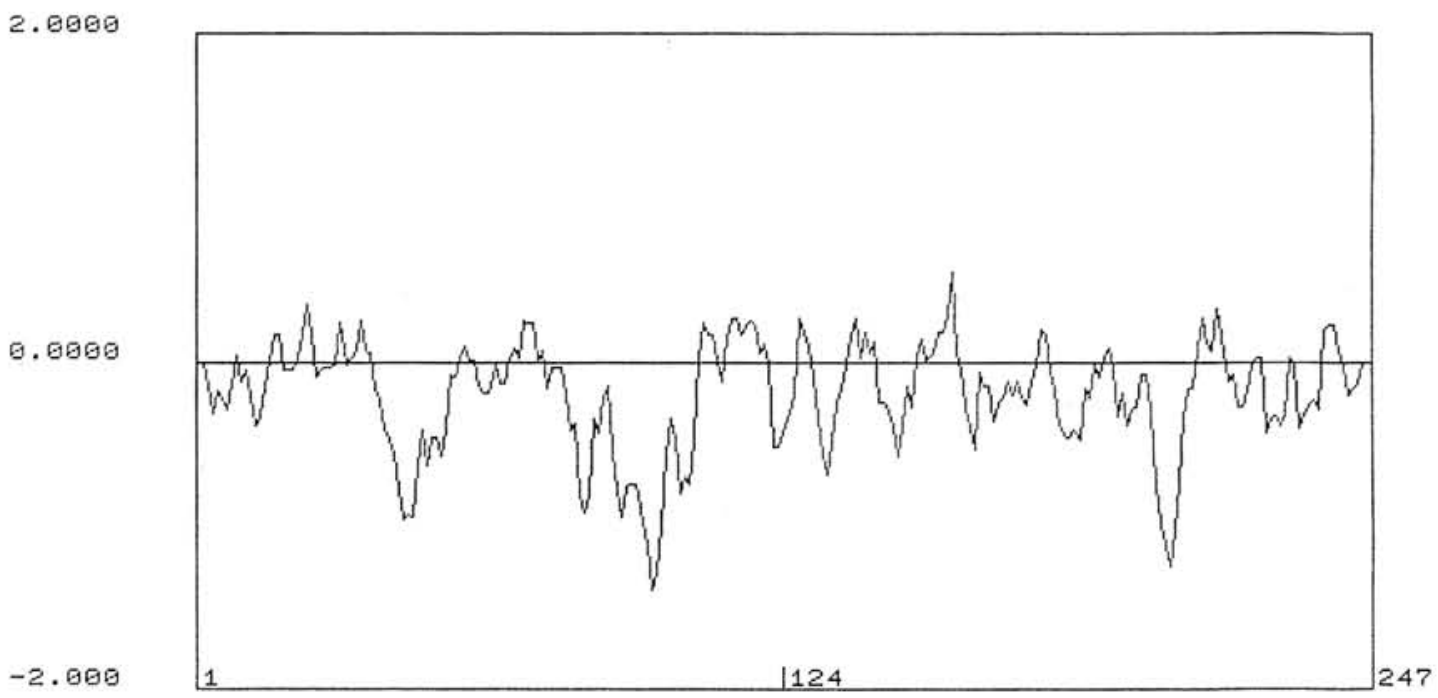

Fig. 3. Hydrophilicity and hydrophobicity plot of putative monkey testis-specific protein. The y axis shows the hydrophilicity and hydrophobicity values $(-2.0000$ to 2.0000$)$ for TSPY as residues shown on the $x$ axis. Values below the 0.0000 mark represent hydrophilic regions; those above are hydrophobic regions. Amino acid positions 42-51, 79-105, and 202-207 show mainly hydrophilicy.

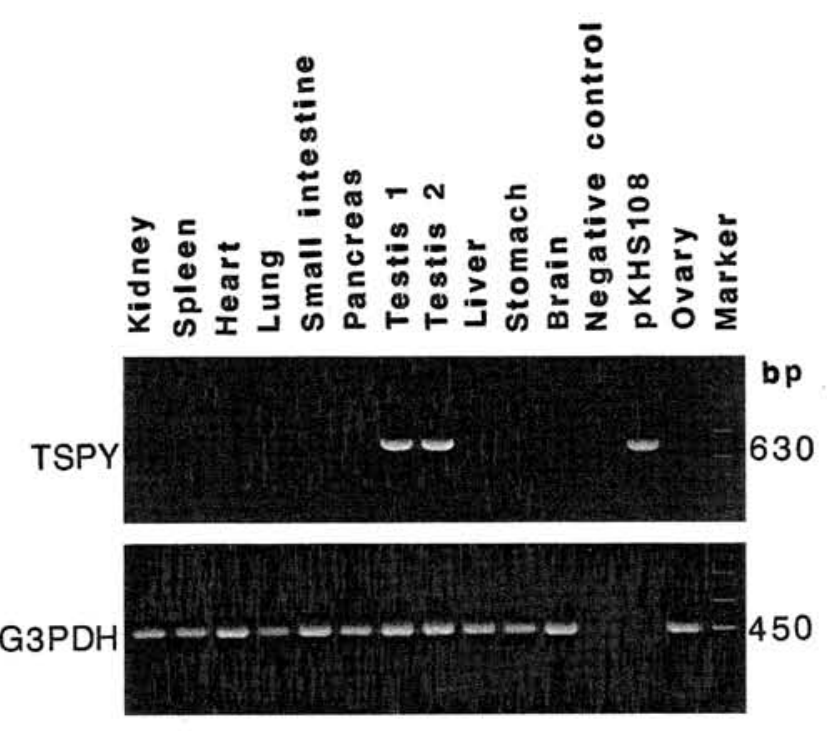

Fig. 4. Expression analyses of the monkey TSPY gene by RT-PCR. The TSPY-specific DNA fragment was amplified by 40 cycles of RTPCR using $1 \mu \mathrm{g}$ each of total RNAs from kidney (lane 1), spleen (lane 2), heart (lane 3), lung (lane 4), small intestine (lane 5), pancreas (lane 6), testes (lanes 7, 8, from different individual sources), liver (lane 9), stomach (lane 10), brain (lane 11), testis (lane 12, without Taq DNA polymerase), and ovary (lane 14). The pKHS108 (TSPY cDNA containing plasmid) was used as positive control (lane 13). As the marker, Taq l-digested pUC118 was loaded in lane 15. The expected fragment size is $630 \mathrm{bp}$ for transcript-specific RT-PCR product. RT-PCR of G3PDH mRNA was used for the quantifications of RNA level in each tissue.

same as that of the first intron of monkey and human TSPY genes cited in our previous report (Kim et al., 1996). In comparison with the coding and $3^{\prime}$ untranslated region of the TSPY gene, $V_{N}$ of the $3^{\prime}$ untranslated region was twofold higher than that of the coding region. Furthermore, comparing the se- quence of the TSPY genomic DNA between great apes and humans, the rates of nucleotide substitutions per site per year were higher in the TSPY intron than in the TSPY exon (Kim and Takenaka, 1996). Therefore, noncoding regions of the TSPY gene evolved more rapidly than the coding region.

We calculated the level of synonymous substitutions per site (Ks) and nonsynonymous substitutions per site (Ka) in the TSPY gene (Table 3). As nonsynonymous substitutions are more strongly influenced by selection than synonymous substitutions, their ratio is a good indicator of selection. Comparing the human-monkey (762 bp) and monkey-bovine (123 bp) TSPY, the values of Ks were 12.6 and 29.8, while those of $\mathrm{Ka} / \mathrm{Ks}$ were 0.68 and 0.84 , respectively. The number of synonymous substitutions were higher than the number of nonsynonymous substitutions in the TSPY gene. Therefore, directional selection has not occurred in the TSPY gene.

\section{RNA expression analyses}

Expression of the TSPY gene was examined by RT-PCR analyses in various tissues including the testis (Fig. 4). Using the specific primers (P355 and P356), a fragment of $630 \mathrm{bp}$ was generated from only testes RNA (lane 7, 8), and no expression was detectable in the other tissues tested. No PCR product was observed in testis without reverse transcriptase treatment (lane 12). When we used plasmid DNA containing monkey TSPY cDNA as a template for PCR, the 630-bp fragment was clearly observed (lane 13). These results showed that the expression of the TSPY gene is specific for testes as suggested by Zhang et al. (1992).

To date, the TSPY, SRY, RNA binding motif (RBM), previously called the YRRM, Y-located RNA recognition motif, and deleted in azoospermia (DAZ) genes are $Y$-specific although there are many homologous genes between the $X$ and $\mathrm{Y}$ chromosomes in man, such as, the ribosomal protein S4 


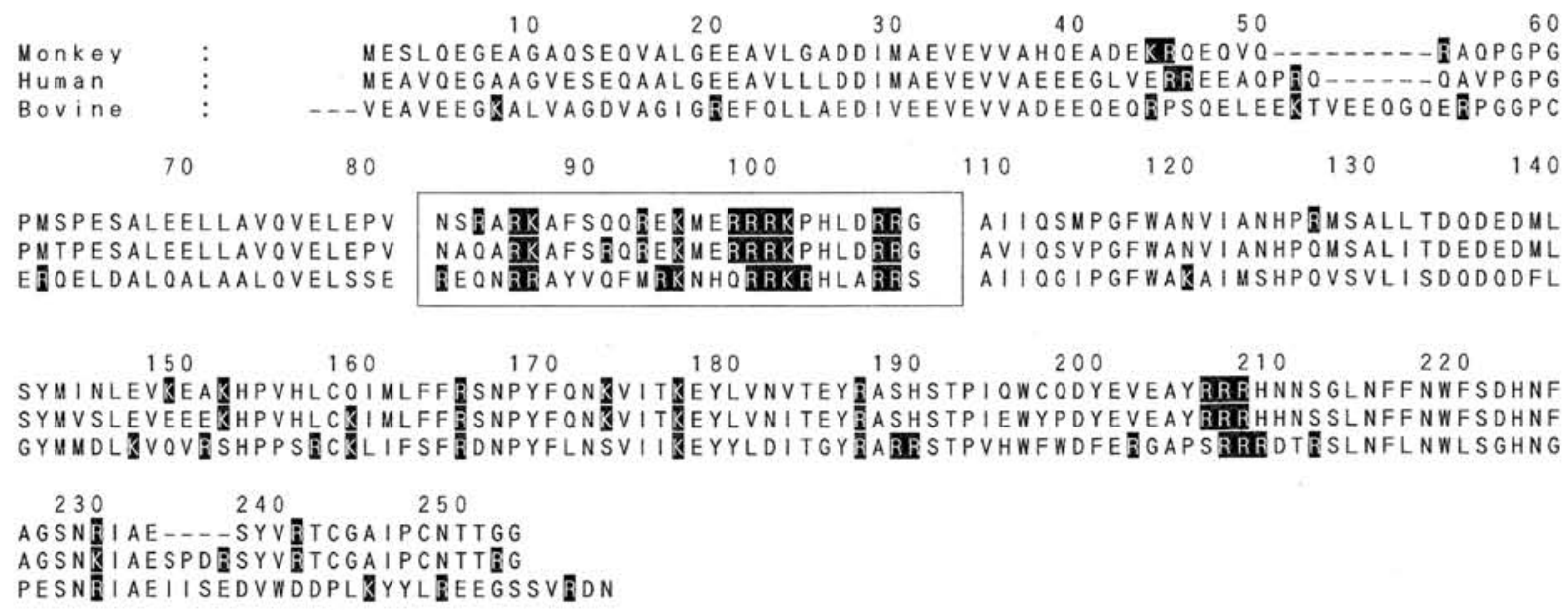

Fig. 5. Comparison of the amino acid sequences of monkey testis-specific protein with those of the human (Zhang et al., 1992) and bovine (Schnieders et al., 1996). The numbering of each residue is based on the human sequence. Although the complete sequence of bovine TSPY has been reported (Schnieders et al., 1996), the $\mathrm{N}$-terminal 53 residues sequence is not shown in Fig. 5 . The nucleotide sequence of the bovine genomic DNA fragment is known (Jakubiczka et al., 1993) and its transcribed amino acid sequence corresponds to residue 107 to residue 147. The nucleotide and deduced amino acid sequences of this fragment were used for comparison with those of monkey and human sequences, respectively. Arg and Lys residues are shown by black background.

Table 1. Percent similarity of nucleotide sequences in the TSPY gene

\begin{tabular}{lcc}
\hline Species pair & Coding region & 3' Untranslated region \\
\hline Human-Monkey & $88.6(762)$ & $76.3(232)$ \\
Human-Bovine & $74.8(123)$ & - \\
Monkey-Bovine & $78.0(123)$ & - \\
\hline
\end{tabular}

The number in parenthesis represent base pairs compared with each other. The same system is used in Table 2 and 3.
Table 2. Mean \pm standard error of the number of nucleotide substitutions per site in the TSPY gene

\begin{tabular}{lccc}
\hline Species pair & \multicolumn{2}{c}{ Coding region } & 3' Untranslated region \\
\hline Human-Monkey & $0.096 \pm 0.012$ & $(762)$ & $0.199 \pm 0.035 \quad(232)$ \\
Human-Bovine & $0.332 \pm 0.070$ & $(123)$ & - \\
Monkey-Bovine & $0.275 \pm 0.060$ & $(123)$ & - \\
\hline
\end{tabular}

Table 3. Synonymous substitutions per site (Ks), nonsynonymous substitutions per site $(\mathrm{Ka})$, and their ratio $(\mathrm{Ka} / \mathrm{Ks})$ in the TSPY gene

\begin{tabular}{llcrc}
\hline Species pair & \multicolumn{2}{c}{$\mathrm{Ks} \times 100$} & $\mathrm{Ka} \times 100$ & $\mathrm{Ka} / \mathrm{Ks}$ \\
\hline Human-Monkey & $12.6 \pm 2.9(762)$ & $8.6 \pm 1.3(762)$ & 0.68 \\
Human-Bovine & $45.1 \pm 17.1(123)$ & $27.4 \pm 6.1(123)$ & 0.61 \\
Monkey-Bovine & $29.8 \pm 12.8(123)$ & $25.0 \pm 5.8(123)$ & 0.84 \\
\hline
\end{tabular}

(RPS4Y), zinc finger protein $Y$ (ZFY), amelogenin (AMELY), and steroid sulphatase (STS-Y). Our results from RT-PCR analysis have demonstrated that the expression of monkey TSPY is confined to the testis. Expression of both TSPY and RBM genes were confined to germ cells of the spermatogonial and early spermatocyte stages of adult human testis in RNA in situ hybridization (Chandley and Cooke, 1994). Testis-specific and germ cell-specific expression of these genes allow us to speculate their specific roles in spermatogenesis.

\section{ACKNOWLEDGMENTS}

This research was supported by Grants-in-Aid for Scientific Research on Priority Areas (No. 06273217 to OT; No. 07640900 to TK) from the Japan Ministry of Education, Science, Sports and Culture, and partially funded by the Sasakawa Scientific Research Grant (No.
7-161 to KHS) from the Japan Science Society.

\section{REFERENCES}

Arnemann J, Jakubiczka S, Thüring S, Schmidtke J (1991) Cloning and sequence analysis of a human $Y$ chromosome derived, tescular cDNA, TSPY. Genomics 11: 108-114

Chandley AC, Cooke HJ (1994) Human male fertility-Y-linked genes and spermatogenesis. Hum Mol Genet 3: 1449-1452

Eisenberg D, Weiss RM, Terwilliger TC (1984) The hydrophobic moment detects periodicity in protein hydrophobicity. Proc Natl Acad Sci USA 81: 140-144

Gingerich PD (1984) Primate evolution: evidence from the fossil record, comparative morphology, and molecular biology. Yearbook Phys Anthropol 27: $57-72$

Guttenbach M, Müller U, Schmid M (1992) A human moderately repeated $\mathrm{Y}$-specific DNA sequence is evolutionary conserved in 
the $Y$ chromosome of the great apes. Genomics 13: 363-367

Holton TA, Graham MW (1991) A simple and efficient method for direct cloning of PCR products using ddT-tailed vectors. Nucl Acids Res 19: 1156

Jakubiczka S, Schnieders F, Schnidtke J (1993) A bovine homologue of the human TSPY gene. Genomics 17: 732-735

Jantzen H-M, Admon A, Bell SP, Tjan R (1990) Nucleolar transcription factor hUBF contains a DNA-binding motif with homology to HMG proteins. Nature 344: 830-836

Kim H-S, Hirai H, Takenaka O (1996) Molecular features in TSPY gene of gibbons and Old World monkeys. Chrom Res 4: 500506

Kim H-S, Takenaka O (1996) A comparison of TSPY genes from Ychromosomal DNA of the great apes and humans: sequence, evolution, and phylogeny. Am J Phys Anthropol 100: 301-309

Kumar S, Tamura K, Nei M (1993) MEGA: Molecular evolutionary genetics analysis, version 1.01. The Pennsylvania State University, University Park, PA 16802

Manz E, Schnieders F, Brechlin AM, Schmidtke J (1993) TSPY-related sequences represent a microheterogeneous gene family organized as constitutive elements in DYZ5 tandem repeat units on the human $Y$ chromosome. Genomics 17: 726-731

Pilbeam D (1986) Distinguished lecture: hominoid evolution and hominoid origins. Am Anthropol 88: 295-312
Sanger F, Nicklen S, Coulson AR (1977) DNA sequencing with chainterminating inhibitors. Proc Natl Acad Sci USA 74: 5463-5467

Schempp W, Binkele A, Arnemann J, Glaser B, Ma K, Taylor K, Toder R, Wolfe J, Zeitler S, Chandley AC (1995) Comparative mapping of YRRM-and TSPY-related cosmids in man and hominoid apes. Chrom Res 3: 227-234

Schnieders F, Dörk T, Arnemann J, Vogel T, Werner M, Schmidtke J (1996) Testis-specific protein, Y-encoded (TSPY) expression in testicular tissues. Hum Mol Genet 11: 1801-1807

Sinclair AH, Berta P, Palmer MS, Hawkins JR, Griffiths BL, Smith MJ, Foster JW, Frischauf A-M, Lovell-Badge R, Goodfellow PN (1990) A gene from the human sex-determining region encodes a protein with homology to a conserved DNA-binding motif. Nature 346: $240-244$

Tajima F, Nei M (1984) Estimation of evolutionary distance between nucleotide sequences. Mol Biol Evol 1: 269-285

Tyler-Smith C, Taylor L, Müller U (1988) Structure of a hypervariable tandemly repeated DNA sequence in the short arm of the human Y chromosome. J Mol Biol 203: 837-848

Zhang JS, Yang-Feng TL, Muller U, Mohandas TK, de Jong PJ, Lau $Y$-FC (1992) Molecular isolation and characterization of an expressed gene from the human $\mathrm{Y}$ chromosome. Hum Mol Genet 1: $717-726$

(Received March 21, 1997 / Accepted April 18, 1997) 